\title{
Cosmological parameter estimation with free-form primordial power spectrum
}

\author{
Dhiraj Kumar Hazra* \\ Asia Pacific Center for Theoretical Physics, Pohang, Gyeongbuk 790-784, Korea \\ Arman Shafieloo ${ }^{\dagger}$ \\ Asia Pacific Center for Theoretical Physics, Pohang, Gyeongbuk 790-784, Korea \\ Department of Physics, POSTECH, Pohang, Gyeongbuk 790-784, Korea \\ Tarun Souradeep $¥$ \\ Inter-University Centre for Astronomy and Astrophysics, Post Bag 4, Ganeshkhind, Pune 411 00\%, India
}

\begin{abstract}
Constraints on the main cosmological parameters using CMB or large scale structure data are usually based on power-law assumption of the primordial power spectrum (PPS). However, in the absence of a preferred model for the early universe, this raises a concern that current cosmological parameter estimates are strongly prejudiced by the assumed power-law form of PPS. In this paper, for the first time, we perform cosmological parameter estimation allowing the free form of the primordial spectrum. This is in fact the most general approach to estimate cosmological parameters without assuming any particular form for the primordial spectrum. We use direct reconstruction of the PPS for any point in the cosmological parameter space using recently modified Richardson-Lucy algorithm however other alternative reconstruction methods could be used for this purpose as well. We use WMAP 9 year data in our analysis considering CMB lensing effect and we report, for the first time, that the flat spatial universe with no cosmological constant is ruled out by more than $4 \sigma$ confidence limit without assuming any particular form of the primordial spectrum. This would be probably the most robust indication for dark energy using CMB data alone. Our results on the estimated cosmological parameters show that higher values of baryonic and matter density and lower value of Hubble parameter (in comparison to the estimated values by assuming power-law PPS) is preferred by the data. However, the estimated cosmological parameters by assuming free form of the PPS have overlap at $1 \sigma$ confidence level with the estimated values assuming power-law form of PPS.
\end{abstract}

PACS numbers:

\section{INTRODUCTION}

The observables of the perturbed universe, such as CMB anisotropy, galaxy surveys and weak lensing, all depend on a set of cosmological parameters assuming a background model describing the current universe, as well as the parameters characterizing the presumed nature of the initial perturbations. While certain characteristics of the initial perturbations, such as the adiabatic nature and tensor contribution, can and are being tested independently, the shape of the PPS remains, at best, a well-motivated assumption. It is important to distinguish between the cosmological parameters within a model that describe the present universe from that characterizing the initial conditions, specifically the PPS, $P(k)$. The standard model of cosmology which is the most popular and widely used cosmological model is the spatially flat $\Lambda \mathrm{CDM}$ model which incorporates a power law form of the primordial power spectrum. The model is described by 6 parameters. Four of them describe the background $\Lambda \mathrm{CDM}$, represented by $\Omega_{b}$ (baryon density), $\Omega_{\mathrm{CDM}}$ (cold dark matter density), $H_{0}$ (present rate of expansion of the universe $)^{1}$ and the reionization optical depth $\tau$. We should mention, dark energy density $\Omega_{\Lambda}$ is directly obtained as a remainder of baryon and cold dark matter density from the total density as we have assumed spatially flat model of the universe. The other two parameters in the model describe the form of the primordial power spectrum which is assumed to be the power law defined by $P(k)=A_{s}\left[\frac{k}{k_{*}}\right]^{n s-1}$ where $A_{s}$ is the amplitude $^{2}$ and the tilt is given by the spectral index $n_{\mathrm{S}}$. The imposed form of the primordial spectrum allows us to provide tight constraints on the four background parameters, however, these tight constraints are basically the result of the rigidness of the model and certain assumption of the primordial spectrum. In other words choosing different assumptions for the form of the primordial spectrum result in different constraints on the background cosmological parameters [1]. In this paper, for the first time we study the complete Markov Chain Monte Carlo parameter estimation assuming a free form of the primor-

\footnotetext{
${ }^{1}$ Sometimes ratio of the sound horizon to the angular diameter distance at decoupling, $\theta$ is considered to be a parameter instead of $H_{0}$

${ }^{2}$ Note that this amplitude is defined at some pivot scale $k_{*}$
} 
dial spectrum through direct reconstruction of the PPS for each point in the background cosmological parameter space using WMAP 9 year data $[2,3]$. There have been different interesting attempts to directly reconstruct the form of the primordial spectrum $[1,4-25]$ and in our analysis we use the recently modified Richardson-Lucy algorithm [25]. We show that it is indeed possible to do the cosmological parameter estimation allowing free form of the primordial spectrum and for the first time we report that a spatially flat universe without cosmological constant is ruled out by more than $4 \sigma$ confidence limit using CMB data alone. This is without putting any prior constraints on the Hubble parameter or using any other cosmological observation. We show that assuming a free form primordial spectrum, the confidence limits of the background cosmological parameters are larger, as expected than those we get by assuming the power-law form of the PPS and we present that the data prefers larger values of baryonic and matter densities for the free form of the primordial spectrum in comparison with power-law assumption. In the next section we discuss the methodology of reconstruction and the parameter estimation followed by which we shall demonstrate our results in the results section. We close with a brief discussion at the end. It should be noted that the aim of this paper is not to relax a parameter of an underlying cosmological model and investigate the effects on other cosmological parameters as usually done to study the cosmographical degeneracies. In this paper we perform cosmological parameter estimation analysis with free form primordial power spectrum which directly indicates that we do not consider any assumptions on underlying inflationary models(or any theoretical model of the early universe). We have been able to compare the free form spectra because of our method [25] is able to identify PPS functions with very large improvement to the WMAP likelihood at any point in cosmological parameter space. This allows estimation of cosmological parameters optimized over the PPS functional degree of freedom.

\section{FORMALISM}

In this work, we have used the recently modified version of Richardson-Lucy algorithm [25] (we call it here after MRL) to reconstruct the optimal form of the primordial spectrum for each point in the background cosmological parameter space. The Richardson-Lucy algorithm [27-30] has been used previously in this context to reconstruct the primordial spectrum $[6,13,14]$ and in the recently modified version one can use the combination of un-binned and binned data in the analysis [25]. The modified algorithm can be formulated as,

$$
\begin{aligned}
P_{k}^{(i+1)}-P_{k}^{(i)} & =P_{k}^{(i)} \times\left[\sum_{\ell=2}^{\ell=900} \widetilde{G}_{\ell k}^{\text {un-binned }}\left\{\left(\frac{C_{\ell}^{\mathrm{D}}-C_{\ell}^{\mathrm{T}(i)}}{C_{\ell}^{\mathrm{T}(i)}}\right) \tanh ^{2}\left[Q_{\ell}\left(C_{\ell}^{\mathrm{D}}-C_{\ell}^{\mathrm{T}(i)}\right)\right]\right\}_{\text {un-binned }}\right. \\
& \left.+\sum_{\ell_{\text {binned }}>900} \widetilde{G}_{\ell k}^{\text {binned }}\left\{\left(\frac{C_{\ell}^{\mathrm{D}}-C_{\ell}^{\mathrm{T}(i)}}{C_{\ell}^{\mathrm{T}(i)}}\right) \tanh ^{2}\left[\frac{C_{\ell}^{\mathrm{D}}-C_{\ell}^{\mathrm{T}(i)}}{\sigma_{\ell}^{\mathrm{D}}}\right]^{2}\right\}_{\text {binned }}\right],
\end{aligned}
$$

Where, $P_{k}^{(i+1)}$ and $P_{k}^{(i)}$ are the power spectrum evaluated in iterations $i+1$ and $i$ respectively and the quantity $\widetilde{G}_{\ell k}$ is normalized radiative transport kernel $G_{\ell k} . C_{\ell}^{\mathrm{D}}$ and $\sigma_{\ell}^{\mathrm{D}}$ are the observed $C_{\ell}^{\mathrm{TT}}$ data points and the corresponding diagonal terms of the inverse covariance matrix. $C_{\ell}^{\mathrm{T}(i)}$ is theoretically calculated angular power spectrum in $i$ 'th iteration. $Q_{\ell}$ is given by the following expression,

$$
Q_{\ell}=\sum_{\ell^{\prime}}\left(C_{\ell^{\prime}}^{\mathrm{D}}-C_{\ell^{\prime}}^{\mathrm{T}(i)}\right) C O V^{-1}\left(\ell, \ell^{\prime}\right)
$$

where $C O V^{-1}\left(\ell, \ell^{\prime}\right)$ is the inverse of the error covariance matrix.

The radiative transport kernel, $G_{\ell k}$ which depends on the background cosmological parameters, satisfies the fol- lowing equation.

$$
C_{\ell}=\sum_{i} G_{\ell k_{i}} P_{k_{i}}
$$

We should mention that as has been indicated in eq. 1 we have used the un-binned data till $\ell=900$ and used the binned data thereafter because of the increasing noise in higher multipoles. For more discussion on this procedure see, ref. [25].

We use publicly available CAMB [31, 32] to calculate the kernel and the $C_{\ell}$ 's and CosmoMC $[33,34]$ to perform the Markov Chain Monte Carlo (MCMC) analysis on the cosmological parameters. Most recent WMAP nine-year observational data [3] has been used in this analysis. We have taken into account the effect of gravitational lensing through approximating the lensing effect on different background models. Basically we assume that for each 
point in the background cosmological parameter space the lensing effect on the observed angular power spectrum would be the same if we assume the power-law PPS or we use the reconstructed PPS. To do so, for each point in the background parameter space one can identify the contribution of gravitational lensing to $C_{\ell}^{\mathrm{TT}}$ 's for the best fit power law primordial spectrum from computed lensing potential power spectrum and using curved-sky correlation function method. We subtract the contribution from the WMAP-unbinned temperature anisotropy data and follow the reconstruction from the modified data. Finally from the reconstructed primordial spectrum we calculate the CMB temperature and polarization $C_{\ell}$ 's and we lens all the $C_{\ell}$ 's again using curved-sky correlation through CAMB [31, 32] and compare them with WMAP data. We should mention that for the multipole range covered by WMAP, the effect of lensing on the temperature and polarization spectrum is not substantial and we find that without lensing too we recover our conclusions of this paper. In order to have a complete analysis we have included the effect of lensing.

We have performed the MRL up to 40 iterations. However we have checked the consistency of our results allowing MRL to work up to different iteration numbers.

\section{RESULTS}

Following the methodology described in the previous section, here we shall discuss the result of our MCMC analysis with the nine year data from WMAP. We find the best fit model provides a $\chi^{2}(-2 \ln \mathcal{L})$ of 7441.4 which is about 115 better than what we get from the best fit power law spectrum. We compare the bounds on the background cosmological parameters with the power law results in figure 1 .

As we are allowing a free form primordial power spectrum, hence having larger effective degrees of freedom, it is expected to have bigger confidence contours compared to the confidence limits derived by assuming power law PPS and fig. 1 clearly illustrates this fact. The results from Fig. 1 suggest that allowing free form PPS, data prefers higher baryon and dark matter densities compared to the canonical results. Best fit values of $\Omega_{\mathrm{b}} h^{2}, \Omega_{\mathrm{CDM}} h^{2}, \mathrm{H}_{0}$ and $\tau$ are $0.0232,0.132,64$ and 0.077 respectively. It should be pointed out that our analysis allows a lower value of optical depth $(\tau)$ which, in turn allows low redshift of reionization $\left(Z_{\mathrm{re}} \sim 7\right)$ compared to the power law results (see last plot of Fig. 1). Our results also indicates that independent of the form of the PPS, a flat model of universe with no cosmological constant is ruled out with a very high confidence. To our knowledge this is the first direct indication towards dark energy with high certainty from CMB temperature and polarization data analysis alone assuming spatial flatness. In figure 2 we plot the one dimensional marginalized likeli- hoods of the parameter $\Omega_{\Lambda}$ obtained using power law (in dashed red line) and allowing free form of the primordial spectrum. The plot clearly demonstrates that a low value of dark energy density is ruled out. Obtained result suggests that values of $\Omega_{\Lambda}<0.25$ is ruled out at $4 \sigma$ which implies a strong exclusion of $\Omega_{\Lambda}=0$ with a very high confidence. In this context it should be noted that no-dark-energy model including curvature was previously ruled out by 3.2- $\sigma$ using Atacama Cosmology Telescope lensing measurements [26] but within the assumption of a power law form of PPS.

In Fig. 3 we plot a few (nearly 100) power spectra (in grey) reconstructed from the WMAP nine-year data with the kernels corresponding to the cosmological parameters within $95 \%(2 \sigma)$ limits of the best fit. We also show the best fit power spectrum from the punctuated inflation model [35] in green and the step models of inflation in blue [36] for comparison. The best fit power law power spectrum is also plotted (in red). We should mention that while in the reconstruction process we have used only temperature data, in the likelihood analysis polarization data is included. As discussed in [25] considering the WMAP polarization data does not significantly improve the reconstruction procedure due to low quality of the polarization data.

We should mention that to check the validity of our approximation regarding lensing contribution, we have repeated our analysis without subtracting the power law lensing effects from the data and we find that the later comparison provides a $\chi^{2}$ worse by 6 than the actual analysis which in turn indicates that our approximation on lensing contribution works well.

To check the robustness of our analysis and the validity of the obtained results, we performed some tests with simulated data. We have synthesized number of $C_{\ell}^{\mathrm{TT}}$ data from angular power spectrum obtained using power law and $\Lambda \mathrm{CDM}$ model with some fixed parameters. With the reconstructed free form spectrum we perform MCMC on the following datasets and calculated the likelihood assuming $\chi^{2}$ distribution ${ }^{3}$. We find that in most of the cases (more than 90\%) the obtained confidence contours of the cosmological parameter contains the fiducial parameter values within the $2 \sigma$ region. This indicates, with the reconstruction we get our fiducial model back in almost all the cases, which, in turn proves the robustness of our analysis.

\footnotetext{
${ }^{3}$ We have shown recently [25] that this likelihood estimator is robust and can be used as an approximation to the complete WMAP likelihood
} 

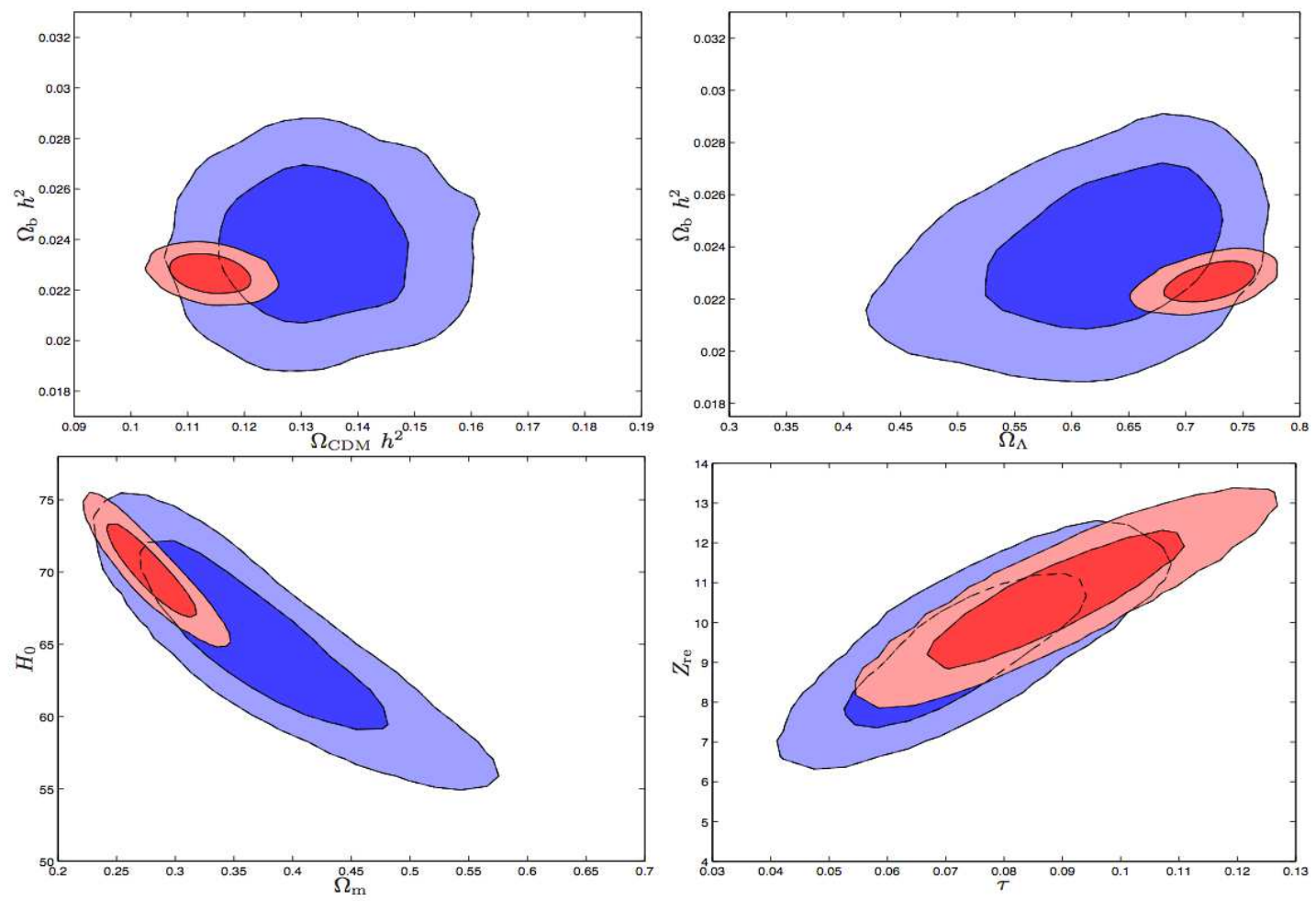

FIG. 1: 2-dimensional confidence contours of the background cosmological parameters. The blue contours represents the results of the analysis allowing free form of the primordial spectrum while the red contours are by assuming the power law form of the primordial spectrum. As expected the free form of the primordial spectrum relaxes the bounds on the parameters. Throughout the analysis we have assumed a spatially flat universe and one can see that a universe with zero density for the cosmological constant is ruled out with high confidence even with no assumption for the primordial spectrum. In comparison with the results from power law assumption of the primordial spectrum, the data prefers higher values of baryonic and matter densities when we allow free form of the primordial spectrum.

\section{DISCUSSION}

In this paper we have estimated the cosmological parameters assuming free form of the primordial spectrum. The primordial spectrum for each point in the background cosmological parameter space is obtained using the MRL reconstruction procedure using the WMAP nine-year combined data of un-binned and binned angular power spectrum. We should mention that for this analysis and instead of MRL one can use trivially other alternative methods of non-parametric reconstruction of the primordial spectrum. In fact the MRL method serves just as a possible method of reconstruction to get a PPS that improves the fit of a cosmological model at different points in the cosmological parameter space to the $\mathrm{CMB}$ data. One is free to choose any other method to do this task. The background model is assumed to be a spatially flat $\Lambda$ CDM model. Performing the MCMC analysis using CosmoMC we obtained the bounds on the background parameters and we find out that the data prefers higher baryonic and matter densities (hence lower $\Omega_{\Lambda}$ ) and lower Hubble parameter when we assume the free form of the
PPS in comparison with the case of power-law assumption. We should mention here earlier efforts [24, 37, 38] have indicated that allowing deviation from simple power law PPS prefers a lower Hubble constant and our result too agrees with that. However, with the ever increasing quality of CMB data from WMAP, we find, our result does not agree with [37] anymore, where it has been shown that allowing deviations from the power law PPS, zero dark-energy model fits the data as well as the $\Lambda \mathrm{CDM}$ model. Our results indicates that independent of the form of the primordial spectrum and without any prior on the value of the Hubble parameter, the spatially flat universe with no cosmological constant is ruled out with a very high confidence using WMAP 9 year data alone. This is the first direct evidence of dark energy with a very high certainty from CMB data alone and with no prior on the Hubble parameter or assuming the form of the primordial spectrum. We expect to get tighter constraints on the background parameters assuming the free form of the primordial spectrum using upcoming Planck data [39]. 


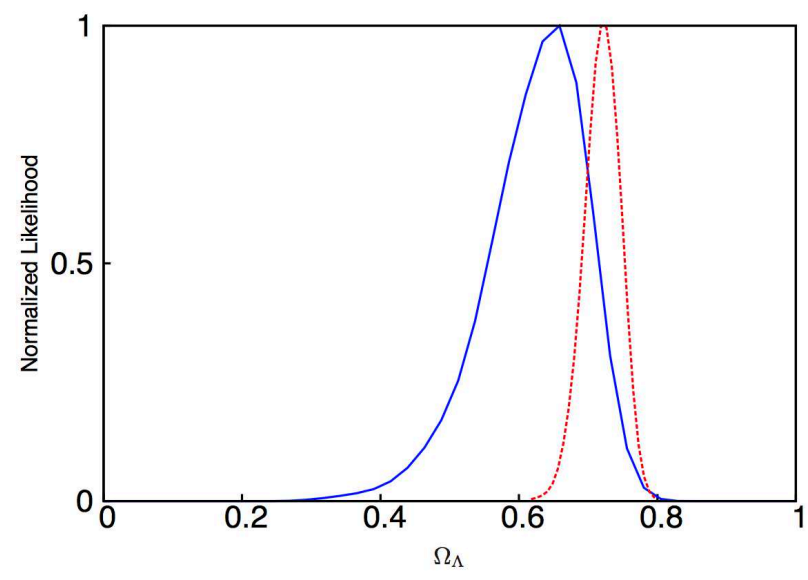

FIG. 2: The one dimensional marginalized likelihood of dark energy density $\Omega_{\Lambda}$ obtained using free form of primordial spectrum (in solid blue line) and using power law (in dashed red line). $\Omega_{\Lambda}=0$ is clearly not favored by the data even if we allow a power spectra free of forms. Quantitatively, in $4 \sigma$ the data rules out $\Omega_{\Lambda}<0.25$. This is probably the first indication towards presence of dark energy with a very high confidence using CMB data alone.

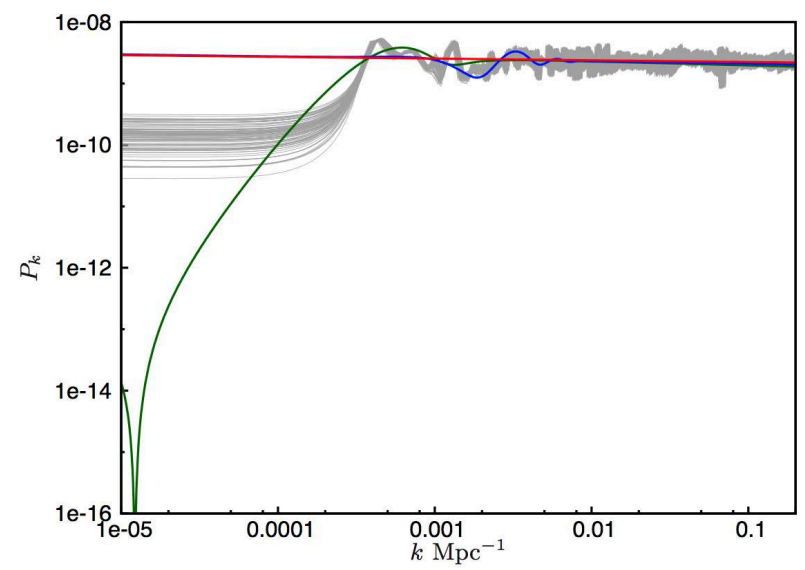

FIG. 3: Reconstructed power spectrum (in grey) obtained from parameters lying within the $2 \sigma$ range of the best-fit likelihood. Over the sample of the reconstructed spectra we have plotted the best fit spectra from the step model [36] (in blue) and the punctuated model [35] (in green) of inflation. The best fit power law power spectrum (in red) is plotted as well for comparison. Note that barring the low- $k$ region (which data has low sensitivity) the sample of reconstructed spectra incorporates nearly all the models within its $2 \sigma$ variation.

Acknowledgments: We would like to thank Teppei Okumura for useful discussions. D.K.H and A.S wish to acknowledge support from the Korea Ministry of Education, Science and Technology, Gyeongsangbuk-Do and Pohang City for Independent Junior Research Groups at the Asia Pacific Center for Theoretical Physics. We also acknowledge the use of publicly available CAMB and CosmoMC to calculate the radiative transport kernel and the angular power spectra. D.K.H would like to acknowledge the use of the high performance computing facilities at the Harish-Chandra Research Institute, Allahabad, India (http://cluster.hri.res.in). TS acknowledges support from Swarnajayanti fellowship grant of DST, India.

* Electronic address: dhiraj@apctp.org

$\dagger$ Electronic address: arman@apctp.org

¥ Electronic address: tarun@iucaa.ernet.in

[1] A. Shafieloo and T. Souradeep, New J. Phys. 13 (2011) 103024 [arXiv:0901.0716 [astro-ph.CO]].

[2] See, http://lambda.gsfc.nasa.gov/product/map/current/.

[3] G. Hinshaw, D. Larson, E. Komatsu, D. N. Spergel, C. L. Bennett, J. Dunkley, M. R. Nolta and M. Halpern et al., arXiv:1212.5226 [astro-ph.CO].

[4] S. Hannestad, Phys. Rev. D 63 (2001) 043009 [astro-ph/0009296].

[5] M. Tegmark and M. Zaldarriaga, Phys. Rev. D 66 (2002) 103508 [astro-ph/0207047].

[6] A. Shafieloo and T. Souradeep, Phys. Rev. D 70 (2004) 043523 [astro-ph/0312174].

[7] S. L. Bridle, A. M. Lewis, J. Weller and G. Efstathiou, Mon. Not. Roy. Astron. Soc. 342 (2003) L72 [astro-ph/0302306].

[8] P. Mukherjee and Y. Wang, Astrophys. J. 599 (2003) 1 [astro-ph/0303211].

[9] S. Hannestad, JCAP $\mathbf{0 4 0 4} \quad$ (2004) 002 [astro-ph/0311491].

[10] D. Tocchini-Valentini, Y. Hoffman and J. Silk, Mon. Not. Roy. Astron. Soc. 367 (2006) 1095 [astro-ph/0509478].

[11] N. Kogo, M. Sasaki and J. 'i. Yokoyama, Prog. Theor. Phys. 114 (2005) 555 [astro-ph/0504471].

[12] S. M. Leach, Mon. Not. Roy. Astron. Soc. 372 (2006) 646 [astro-ph/0506390].

[13] A. Shafieloo, T. Souradeep, P. Manimaran, P. K. Panigrahi and R. Rangarajan, Phys. Rev. D 75 (2007) 123502 [astro-ph/0611352].

[14] A. Shafieloo and T. Souradeep, Phys. Rev. D 78 (2008) 023511 [arXiv:0709.1944 [astro-ph]].

[15] R. Nagata and J. 'i. Yokoyama, Phys. Rev. D 78 (2008) 123002 [arXiv:0809.4537 [astro-ph]].

[16] T. Souradeep and A. Shafieloo, Prog. Theor. Phys. Suppl. 172 (2008) 156.

[17] R. Nagata and J. 'i. Yokoyama, Phys. Rev. D 79 (2009) 043010 [arXiv:0812.4585 [astro-ph]].

[18] K. Ichiki and R. Nagata, Phys. Rev. D 80 (2009) 083002.

[19] P. Paykari and A. H. Jaffe, Astrophys. J. 711 (2010) 1 [arXiv:0902.4399 [astro-ph.CO]].

[20] G. Nicholson and C. R. Contaldi, JCAP 0907 (2009) 011 [arXiv:0903.1106 [astro-ph.CO]].

[21] G. Nicholson, C. R. Contaldi and P. Paykari, JCAP 1001 (2010) 016 [arXiv:0909.5092 [astro-ph.CO]].

[22] M. Bridges et al, Mon. Not. R. Astron. Soc. $4001075 B$ (2009)

[23] C. Gauthier and M. Bucher, JCAP 1210 (2012) 050 
[arXiv:1209.2147 [astro-ph.CO]].

[24] R. Hlozek, J. Dunkley, G. Addison, J. W. Appel, J. R. Bond, C. S. Carvalho, S. Das and M. Devlin et al., Astrophys. J. 749 (2012) 90 [arXiv:1105.4887 [astroph.CO]].

[25] D. K. Hazra, A. Shafieloo and T. Souradeep, JCAP 1307, 031 (2013) [arXiv:1303.4143 [astro-ph.CO]].

[26] B. D. Sherwin, J. Dunkley, S. Das, J. W. Appel, J. R. Bond, C. S. Carvalho, M. J. Devlin and R. Dunner et al., Phys. Rev. Lett. 107, 021302 (2011) [arXiv:1105.0419 [astro-ph.CO]].

[27] B. H. Richardson, J. Opt. Soc. Am. 6255 (1972)

[28] L. B. Lucy, Astron. J. 796 (1974)

[29] C. M. Baugh and G. Efstathiou, Mon. Not. R. Astron. Soc. 265145 (1993)

[30] C. M. Baugh and G. Efstathiou, Mon. Not. R. Astron. Soc. 267323 (1994)

[31] See, http://camb.info/.

[32] A. Lewis, A. Challinor and A. Lasenby, Astrophys. J. 538 (2000) 473 [astro-ph/9911177].
[33] See, http://cosmologist.info/cosmomc/.

[34] A. Lewis and S. Bridle, Phys. Rev. D 66 (2002) 103511 [astro-ph/0205436].

[35] R. K. Jain, P. Chingangbam, J. -O. Gong, L. Sriramkumar and T. Souradeep, JCAP 0901 (2009) 009 [arXiv:0809.3915 [astro-ph]]

[36] A. A. Starobinsky, JETP Lett. 55 (1992) 489 [Pisma Zh. Eksp. Teor. Fiz. 55 (1992) 477]; J. A. Adams, B. Cresswell and R. Easther, Phys. Rev. D 64 (2001) 123514 [astro-ph/0102236]; D. K. Hazra, M. Aich, R. K. Jain, L. Sriramkumar and T. Souradeep, JCAP 1010 (2010) 008 [arXiv:1005.2175 [astro-ph.CO]].

[37] A. Blanchard, M. Douspis, M. Rowan-Robinson and S. Sarkar, Astron. Astrophys. 412, 35 (2003) [astro-ph/0304237].

[38] P. Hunt and S. Sarkar, Phys. Rev. D 76, 123504 (2007) [arXiv:0706.2443 [astro-ph]].

[39] See, http://www. sciops.esa.int/PLANCK/. 